\title{
ApOFIS: an A priori Optical Font Identification System
}

\author{
Abdelwahab Zramdini and Rolf Ingold \\ Institute of Informatics, University of Fribourg \\ Chemin du Musée 3, CH-1700 Fribourg (Switzerland) \\ E-mail: abdelwahab.zramdini@unifr.ch http://www-iiuf.unifr.ch/GIRAF/giraf.html
}

\begin{abstract}
The detection of the font style, point size, etc. of a text is an obvious way to improve the capabilities of text recognition algorithms. The ApOFIS system has been designed in order to satisfy such a requirement. It adopts an a priori font identification approach where the recognition of a text font is done without considering the characters that appear in the text. In ApOFIS, a font is characterized especially by its family, weight, slope and size. Features used in the system represent global aspects of text line images. They have been extracted essentially from projection profiles and from connected components bounding boxes. Statistical tests have revealed that these features follow approximately normal laws so that parameter estimation is used in learning.

A multivariate Bayesian classifier, based on these features, has been designed for font recognition and applied on a base of 240 font models created from a training set of texts written with thęse fonts. On text lines having the same length as those used for learning, the system allows to discriminate fonts with an average accuracy of $96.5 \%$ for top choice and $98.3 \%$ within the two top choices.
\end{abstract}

Keywords: Document Analysis, a priori Font Recognition, Bayesian Classifier

\section{Introduction}

The SSPR'90 working group on Character Recognition stated that [1]:

The detection of the font style, point size, etc. of a text is an obvious way to improve the capabilities of text recognition algorithms. This would allow for hundreds of fonts to be used for training but retain the recognition accuracy and potential speed of a system that uses a small number of fonts. This appears to be a promising but hitherto almost neglected topic.

In this context, the Optical Font Recognition (OFR) become an important topic in optical reading. Furthermore, OFR is gaining some interest during the last few years $[1,2,3]$. Indeed, there are two possible approaches for font recognition:

1. a priori font recognition where fonts are identified without considering the characters that appear in the text. Thus, features have to be extracted from global aspects of the text images. These aspects are generally detected by non experts in typography such as the text density, letters size, orientation and spacing, and the presence of serifs. Features may be computed from different text entities such as words or lines.

2. a posteriori font recognition taking a substantial benefit from the knowledge of the letter classes. Thus, features can be based on local aspects of individual letters or their particularities such as the shape of serifs (cove, squared, triangular, etc.) and the representation of particular letters like $\mathrm{g}$ and $\mathrm{g}$, a and $a$, etc. 
In the context of a structured document recognition system developed in our laboratory [4], font identification plays an important role. For instance, an a priori font recognition approach has been adopted and evaluated through the ApOFIS system.

In our OFR system, a font is defined by five attributes: family (Times, Helvetica, etc. $)^{1}$, size expressed in typographical points, weight (regular, bold), slope (roman, italic) and width (normal, expanded, condensed).

The goal of the present paper is to present the ApOFIS system and an evaluation of its performances.

\section{ApOFIS Architecture}

ApOFIS is based on a Bayesian classification model and uses nine global features extracted from text line images.

\subsection{Selected features}

Three features are extracted from the vertical projection profile $V_{p}$ (see Fig. 1):

- $h w p$ : height of the whole vertical profile;

- hup: height of the upper part of the vertical profile;

- $h c p$ : height of the central part of the vertical profile.

These heights simulate the typographical notions of font-height, X-height and $x$-height. Two other features are extracted from the horizontal projection profile $H_{p}$ (see Fig. 1):

- $d n$ : the density of black pixels in a rectangular box around the considered text line. Indeed, the weight of a font is reflected by the density of black surfaces on the white background. If $n$ is the size of $H_{p}$, then this density is defined by

$$
d n=\frac{1}{n} \sum_{i=1}^{n} H_{p}[i]
$$

$-p d^{2}$ : the means of the squared values of the horizontal profile first derivative. One can observe from $H_{p}$ that roman texts are characterized by a set of upright and tall peaks. For italic texts the peaks are less tall, rounded and boarder. Let $n$ be the size of $H_{p}$, this feature is defined by

$$
p d^{2}=\frac{1}{n-1} \sum_{i=1}^{n-1}\left(H_{p}[i+1]-H_{p}[i]\right)^{2} .
$$

Two other features are extracted from connected components and especially from their bounding boxes (see Fig. 2). Let $d\left(e_{i}, e_{i+1}\right)$ be the horizontal distance in pixels between two consecutive connected components bounding boxes and let $w\left(e_{i}\right)$ be the width of the connected component $i$ bounding box. Let us suppose a text line with $n$ connected components, the following features have been defined:

\footnotetext{
${ }^{1}$ Commonly, the family attribute corresponds to the typographical notion of "typeface".
} 


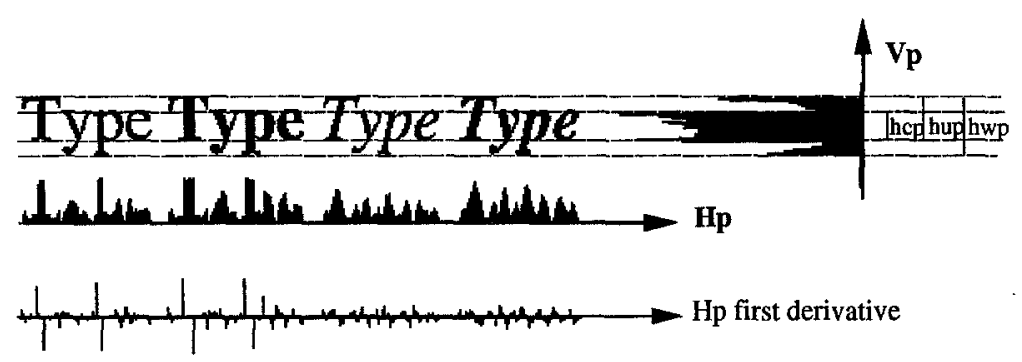

Fig. 1. Projection profiles and first derivative of the horizontal profile.

- ccsp: the means of connected components spacing within text words, defined by

$$
c c s p=\frac{1}{n-1} \sum_{i=1}^{n-1} d\left(e_{i}, e_{i+1}\right) .
$$

As shown by Fig. 2, characters spacing within the same word changes from one font to another. One notices that this spacing is very important and variable for fixed pitch fonts. It is also more important for sans-serif fonts than for seriffed ones;

- $c c w$ : the average width of connected components bounding boxes in the text line, defined by

$$
c c w=\frac{1}{n} \sum_{i=1}^{n} w\left(e_{i}\right) .
$$

This feature is relevant especially for connected components with squared forms corresponding to main characters in the central zone of the vertical profile such as characters $a, c, n$ of the word Spacing. Indeed, these character widths depend on the font family, weight and size.
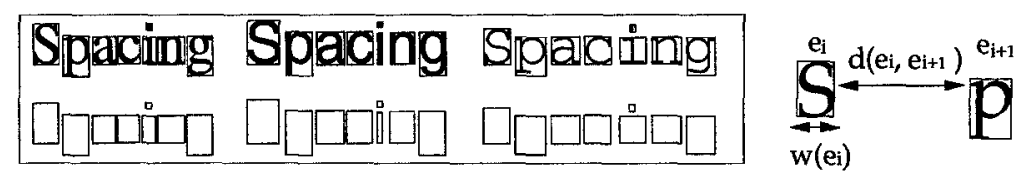

Fig. 2. Connected components bounding boxes spacing and widths.

Finally, two other features are extracted from special zones in the text image:

- hrl: the average width of the vertical stems within characters which depends on the font family, weight and size (see Fig. 3(a)). The stem widths are estimated by computing the horizontal run-lengths within the text image.

Let $h r l_{i}$ be the length of a horizontal run $i$ and $n$ be the amount of runs in the text, this feature is defined by

$$
h r l=\frac{1}{n} \sum_{i=1}^{n} h r l_{i}
$$

Since it considers all characters, $h r l$ includes other information than stems such as arcs of characters $o$ and $c$. 
- pdif: the density of character contours computed on particular regions around the baseline and the upperline as shown by Fig. 3(b). Indeed, serifs which distinguish between seriffed and sans-serif fonts, are located at the end of character main strokes, especially around the baseline. Practically, the contour is detected by computing the difference between consecutive horizontal pixel lines in the text image.

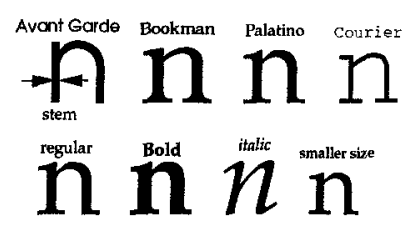

Fig. 3. (a) Stems width for various styles

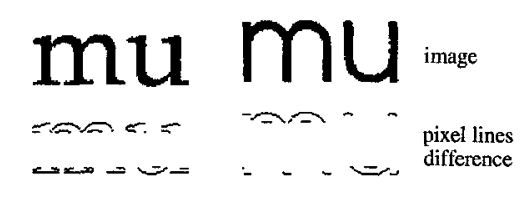

(b) pixel lines difference.

\subsection{Classification model}

APOFIS is based on a Bayesian classification model where the font models base used in the classification is created from a training set of text line images. We have shown in [5] that we can assume that features follow normal laws, thus the learning process consists in estimating the multivariate normal density parameters $(\mu, \Sigma)$ where $\mu$ expresses the means features vector and $\Sigma$ the corresponding covariance matrix.

Learning The learning allows to create a font models base from samples of the considered fonts. Learning a font $\left(f_{i}\right)$ consists in:

1. generating an image by scanning a text page printed with $f_{i}$;

2. performing some preprocessing on the image in order (1) to detect and correct possible skew and (2) to filter noise, punctuation and diacritical signs which do not carry any pertinent information;

3. segmenting the image into lines;

4. extracting a features vector $x=\left(x_{1}, \ldots, x_{n}\right)$ from each line (in our case $n=9$ );

5. collecting these vectors to estimate $\mu$ and $\Sigma$ by the maximum likelihood estimator;

6. finally, adding the font model $\left(\mu, \Sigma, f_{i}\right)$ to the base.

The current font models base of ApOFIS system contains 240 font models corresponding to 15 typefaces (see Table 1), four sizes $(10,11,12,14 \mathrm{pt}$ ) and four styles (regular, italic, bold, bold-italic) often used in documents. Therefore, 16 fonts have been considered for each typeface. For each font, $\mu$ and $\Sigma$ have been computed on features extracted from 100 English text lines of about $6 \mathrm{~cm}$ length. Texts have been arbitrarily extracted from existing documents and produced by a laser printer. Binary images have been produced from these texts by a scanning at $400 \mathrm{dpi}$.

Classification The goal of the classification process is to assign a font to a text line modeled by a features vector. The classification consists in traversing the font models base and associating to this vector the font model which maximizes a certain function. This function measures the probability that this vector corresponds to the font model[2]. 


\section{Evaluation of the System Performances}

\subsection{Methodology}

For each font, the classification has been done on 100 French text lines with almost the same length as those used for learning. Texts have been produced under the same conditions as during learning: they have been printed with the same printer and scanned using the same scanning parameters (thresholds, resolution, etc.). The classification has been performed by a multivariate Bayesian classifier using the base of 240 font models.

The algorithm is applied on each image (corresponding to a text written with one font) such that for each text line, the classifier returns in a decreasing order the most probable font identifiers. A font is considered to be correct if its family, weight, slope and size are correct. A simple averaging is then performed and a font recognition rate is retained for the whole image. Other average recognition rates are computed for individual font attributes, i.e. the family, weight, slope and size.

\subsection{Results}

Table 1 shows the average recognition rates for fonts and font attributes. Fonts have been quite well recognized with an average rate of $96.5 \%$ for top choice, $98.3 \%$ within the two top choices. These rates reach an accuracy of $99 \%$ within the four top choices. Weights and slopes have been accurately recognized with rates above $99 \%$, while families and sizes recognition has been less accurate with an average rate of $97 \%$.

The experience has shown the presence of three typeface clusters (seriffed, sans-serif and fixed pitch), such that family misclassifications mainly occur within the same cluster. In practice, a $99 \%$ discrimination rate between seriffed and sans-serif fonts has been reached, which can be very helpful for OCR systems.

Table 1. Average recognition rates of fonts and font attributes performed by a Bayesian classifier applied on 240 fonts using a base of 240 font models (1): top choice, (2): two top choices].

\begin{tabular}{|l|l|l||l|l|l|l|}
\hline & Font (2) & Font (1) & Family(1) & Weight(1) & Slope(1) & Size(1) \\
\hline Avant Garde & 0.996 & 0.990 & 0.991 & 0.999 & 0.998 & 0.996 \\
Bookman & 0.985 & 0.971 & 0.973 & 0.999 & 0.999 & 0.989 \\
Computer Modern & 0.957 & 0.913 & 0.981 & 0.999 & 0.999 & 0.934 \\
Courier & 0.970 & 0.945 & 0.991 & 0.999 & 0.997 & 0.929 \\
Franklin Gothic & 0.997 & 0.980 & 0.980 & 0.999 & 0.997 & 0.991 \\
Garamond & 0.995 & 0.992 & 0.994 & 0.999 & 0.999 & 0.995 \\
Helvetica & 0.982 & 0.967 & 0.967 & 0.999 & 0.999 & 0.990 \\
Lubalin Graph & 0.966 & 0.960 & 0.964 & 0.999 & 0.995 & 0.974 \\
Lucida Bright & 0.996 & 0.987 & 0.991 & 0.999 & 0.997 & 0.994 \\
Lucida Sans & 0.996 & 0.964 & 0.967 & 0.999 & 0.998 & 0.991 \\
Lucida Sans TypeW. & 0.995 & 0.983 & 0.985 & 0.999 & 0.998 & 0.993 \\
New Century Schlbk & 0.997 & 0.980 & 0.984 & 0.998 & 0.999 & 0.991 \\
Palatino & 0.980 & 0.959 & 0.960 & 0.997 & 0.998 & 0.983 \\
Souvenir & 0.982 & 0.955 & 0.957 & 0.996 & 0.997 & 0.986 \\
Times & 0.959 & 0.924 & 0.959 & 0.998 & 0.993 & 0.956 \\
\hline average & 0.983 & 0.965 & 0.976 & 0.998 & 0.997 & 0.979 \\
\hline
\end{tabular}


The approach seems very promising in the context of real document structures recognition because:

- generally, the fonts used in the document is known a priori, which allows to reduce the size of the font models base;

- typographers use few and very distinct typefaces within the same document.

\section{Conclusions}

We have shown in this paper the reliability of an a priori font recognition approach based on statistical analysis of text line images. Some evaluations have shown the system efficiency when images used in the tests are produced under the same conditions as those used for training. Indeed, the system allows an accurate identification of fonts and their individual attributes. In order to improve the system usability, other quantitative evaluations have to be carried out.

The first one consists in evaluating the system behavior in the context of degraded images. We have shown in [6] that the system is relatively robust against natural degradations generated by scanners and photocopiers but its performances drop dramatically in presence of very degraded images. In order to improve the system tolerance to degradations, an approach allowing an automatic adaptation of the system to modeled degradations, has been adopted and provided some promising results.

The second one aims at the study of the effects of the text length and content on the system performances. Preliminary evaluations of ApOFIS on single words have shown that it performs an accurate font style (weight, slope) recognition on words of more than 3 letters. We are currently, analyzing how well font models computed from text lines (set of words) can be adapted to recognize single words.

\section{References}

1. T. Bayer, J. Hull, and G. Nagy, 'Character Recognition: SSPR'90 working group report', in Structured Document Image Analysis, eds., H.S. Baird, H. Bunke, and K. Yamamoto, 567567, Springer Verlag, (1992).

2. A. Zramdini and R. Ingold, 'Optical font recognition from projection profiles', in RIDT'94: Third International Conference on Raster Imaging and Digital Typography, pp. 249-260, Darmstadt, Germany, (4 1994).

3. R. A. Morris, 'Classification of digital typefaces using spectral signatures', Pattern Recognition, 25(8), 869-876, (1988).

4. Tao Hu, New Methods for Robust and Efficient Recognition of the Logical Structures in Documents, Ph.D. dissertation, University of Fribourg, 1994.

5. A. Zramdini and R. Ingold, 'A priori font recognition using a Bayesain classifier', Technical report, IIUF, University of Fribourg, Switzerland, (2 1994).

6. A. Zramdini and R. Ingold, 'A Study of Document Image Degradation Effects on Font Recognition', to be published in ICDAR'95: Third International Conference on Document Analysis and Recognition, Montreal, Canada, (8 1995). 